\title{
Multicenter survey on emergency nurses' perception of Numerical Rating Scale reliability at triage time in adult Emergency Department patients
}

\author{
Gianfranco Cervellin, ${ }^{1}$ Ivan Comelli, ${ }^{1}$ Andrea Bellone, ${ }^{2}$ Fabrizio Giostra, ${ }^{3}$ Nicoletta Acierno, ${ }^{4}$ \\ Michele Mitaritonno, ${ }^{5}$ Ciro Paolillo, ${ }^{6}$ Fabrizio Mastroianni, ${ }^{7}$ Matteo Pistorello, ${ }^{8}$ Gianni Rastelli, ${ }^{9}$ \\ Giorgio Ricci, ${ }^{10}$ Andrea Tenci, ${ }^{11}$ Guido Caironi ${ }^{12}$ \\ ${ }^{1}$ Emergency Department, University Hospital of Parma, Parma; ${ }^{2}$ Emergency Department, Niguarda \\ Hospital, Milan; ${ }^{3}$ Emergency Department, Hospital of Fermo, Fermo $;{ }^{4}$ Emergency Department, S. Paolo \\ Hospital, Savona; ${ }^{5}$ Emergency Department, Hospital of Cosenza, Cosenza; ${ }^{6}$ Emergency Department, Spedali \\ Riuniti University Hospital, Brescia; ${ }^{7}$ Emergency Department, Hospital of Aosta, Aosta; ${ }^{8}$ Emergency \\ Department, Hospital of Treviso, Treviso; ${ }^{9}$ Emergency Department, Hospital of Vaio, Fidenza; ${ }^{10}$ Emergency \\ Department, University Hospital of Verona, Verona; ${ }^{11}$ Emergency Department, Girolamo Fracastoro \\ Hospital, San Bonifacio (VR); ${ }^{12}$ Emergency Department, Local Health Agency, Como, Italy
}

\begin{abstract}
Since pain perception is highly subjective and culturally mediated, its objective evaluation remains difficult. Nevertheless, pain measurement should ideally be a part of the assessment of patients in order to plan adequate pain relief. Several scales have been proposed for pain measurement, being the numerical rating scale (NRS) the most widely used, often at triage time. NRS have demonstrated acceptable reliability and validity, in post-operative medicine and in oncologic pain, but data in the Emergency Departments (EDs) are poor. The aim of this study is to evaluate the Emergency Nurses' (ENs) perception about the reliability of
\end{abstract}

\footnotetext{
Correspondence: Gianfranco Cervellin, Emergency Department, University Hospital of Parma, Via Gramsci 1443126 Parma, Italy.

E-mail: gcervellin@ao.pr.it

Key words: Pain; Numeric rating scale; Triage; Emergency department

Acknowledgements: the authors acknowledge all the participating nurses of the involved EDs, for having kindly responded to the questionnaire. They also acknowledge Prof. Alessandra Cassar, Department of Economics, University of San Francisco, for the help with the statistical analysis
}

Contributions: GC1 and IC conceived the study and made the questionnaire; all the Authors contributed to data collection; GC12 coordinated the data collection; $\mathrm{GC} 1$ wrote the manuscript; all the Authors reviewed the manuscript.

Conflict of interest: the authors declare no potential conflict of interest.

Funding: none.

Received for publication: 27 August 2018.

Revisions received: 2 October 2018.

Accepted for publication: 30 October 2018.

This work is licensed under a Creative Commons Attribution 4.0 License (by-nc 4.0)

(C) Copyright G. Cervellin et al., 2018

Licensee PAGEPress, Italy

Emergency Care Journal 2018; 14:7787

doi:10.4081/ecj.2018.7787
NRS in the triage process. A questionnaire based on 11 items was designed and subsequently administered to a large number of ENs in several EDs in Northern and Central Italy. 301 questionnaires were filled out and returned. The majority declares using NRS scale to measure pain (item 2 , mode $=4$, mean $=3.8$ ), and attributing priority code based on NRS value (item 3 , mode $=4$, mean $=$ 3.4). In general, triage nurses believe that NRS is only indicative and that their judgement matters (item 4 , mode $=4$, mean $=3.2$ ). The vast majority of triage nurses do believe that the patients will indicate a fake higher NRS value with the aim to get a more urgent code (item 5 , mode $=5$, mean $=4$ ), while only a small minority expects that patients would underestimate their NRS for fear of penalizing more urgent patients. Very few believe that such scale underestimates the patients' condition, while the majority is ambivalent about whether such scale overestimates it. In conclusion, NRS confirms to be a potentially valuable tool for pain evaluation at triage time, but many nurses express some doubts on its reliability, and will attribute the triage code mainly basing on their own judgement.

\section{Introduction}

The culture of health-parameters measurement appeared only recently in the history of medicine. This effort is not merely an intellectual exercise: the selection of particular health indicators reflects the perceived importance of a problem at a particular historical period. Hence, the results of these measurements commonly become tools for the organizations promoting health policies. One classic definition of measurement is "the assignment of numbers to objects or events to represent quantities of attributes according to rules". ${ }^{1}$ Hence, the process of measuring health parameters needs standardized questions about a particular condition, and require the assignment of numbers to each answer. These numbers may then be aggregated into a cumulative score, intended to reflect some aspect of health. When measuring any biological variable, the pivotal questions should be: "Does this instrument measure what it is expected to measure?", and "Is the parameter I'm measuring distributed in the population according to the Gauss' law?". These two questions deal with validity and reliability (namely, the replicability of an instrument's results) of the chosen tool. Pain, one of the leading symptoms of presentation to the Emergency Department (ED), should not be considered an exception. Since pain perception is highly subjective and culturally mediated, however, its 
objective evaluation remains difficult, and the validity of patient self-report is often questioned. ${ }^{2-4}$ Nevertheless, pain measurement should ideally be a part of the assessment of patients in order to plan the most appropriate therapeutic management, namely adequate pain relief. ${ }^{5}$ Some Authors have proposed that pain represents the fifth (or sixth, when considering peripheral oxygen saturation) vital sign, thus compelling nurses and physicians to consider the evaluation of its intensity as part of patient assessment and documentation. ${ }^{6}$ It is, however, of pivotal importance to understand that pain is an intimate experience, and neither blood tests, tissue pathology, diagnostic imaging, physical assessments, nor patient behaviours reliably reflect the true pain experience.

Several scales have been proposed in literature for pain measurement. For practical purposes, they should be easy to use, have clear data recording and processing, and fulfil criteria of validity, sensitivity and reliability. The four main tools presently used to rate pain intensity are: i) the numerical rating scale (NRS), ii) the visual analog scale (VAS), iii) the graphical rating scale (GRS), and iv) the verbal rating scale (VRS). In the majority of western countries, notably in adult patients, in most EDs various form of NRS are routinely used, often at triage time. ${ }^{5}$ NRS consists of a range of numbers, usually from 0 to 10 , from which one is selected by the patient as most representative of his/her level of pain intensity. In this range, zero represents no pain and 10 represents some maximal measure of pain, like pain as intense as you can imagine or worst pain possible. NRS have demonstrated acceptable reliability and validity, especially in post-operative medicine and in oncologic pain, ${ }^{7-12}$ but data in the EDs are poor. Nevertheless, in many Italian EDs NRS contributes to the attribution of the triage code, thus establishing the priority to the first medical contact. Many patients are aware of this, and recently some concerns have been raised about the possibility of them adopting some forms of simulation with the goal to achieve a higher triage code, and, consequently, a shorter length of stay in the ED. As such, the aim of this study is to evaluate the Emergency Nurses' (ENs) perception about the reliability of NRS in the triage process.

\section{Materials and Methods}

\section{Design}

A questionnaire based on 11 items/statements (Table 1) was designed by a group of members of our scientific society - the Academy of Emergency Medicine and Care (AcEMC) - and subsequently administered either directly (by a physician or by the head of nurses) or via e-mail to a large number of ENs in several Italians EDs. The nurses were informed that the questionnaire was anonymous, and they were free to participate or not.

\section{Data collection}

The questionnaire asks about pain measurement at triage time in adult patients only (i.e., aged more than 14 years). Some additional information, such as the respondent's age, gender, and years of work as triage nurse were obtained. The responses were collected in an anonymous way. For each center, a physician or a nurse was responsible for the distribution and collection of the questionnaires, as well as for returning the surveys to our administrative assistant. All the questionnaires were filled out and collected between May $1^{\text {st }}$ and June $15^{\text {th }}, 2018$.

The study was performed in agreement with the ethical standards established by the institution in which the experiments were performed and the Helsinki Declaration of 1975. Due to the intrinsic nature of the study, an approval by ethic committee was not required. Each nurse freely decided to respond or not to the questionnaire.

\section{Statistical analysis}

The statistical analysis was performed using Stata/SE $15.1^{\circledR}$. The mean values \pm Standard Deviation (SD) of the respondents' rating of their agreement or disagreement with each of the eleven statements are reported in Table 2. The linear regressions for each item $\mathrm{Y}$ was performed according to the following formula:

$\mathrm{Y}_{\mathrm{i}}=\beta 0+\beta 1$ Age $_{\mathrm{i}}+\beta 2$ Gender $_{\mathrm{i}}+\beta 3$ Experience $_{\mathrm{i}}+\beta 4$ Triage $_{\mathrm{i}}+\varepsilon_{\mathrm{i}}$

Table 1. The questionnaire.

$\begin{array}{cccc}1 & 2 & 3 & 4 \\ \text { Totally } & \text { Partially } & \text { Neither agree Partially Totally } \\ \text { disagree disagree } & \text { nor disagree }\end{array}$

1 I consider essential to measure, not only to evaluate, patients' pain at triage.

2 I always measure patients' pain using NRS.

3 I attribute the triage code based on NRS results.

4 NRS results are only approximate. I decide the triage code based on my personal judgement.

5 Several patients declare a high NRS value with the aim to achieve a higher triage code.

6 Several patients declare a low NRS value due to the worry to penalize patients with worse problems.

7 I believe to give right triage codes, appropriate to the suffering condition of the patients.

8 Sometimes I overrate triage codes to avoid conflicts and problems.

9 Sometimes I underrate triage codes to avoid ED overload.

10 By using NRS there is a risk of underestimate patients' problems.

11 By using NRS there is a trend toward overestimate patients' problems.

NRS, numerical rating scale; ED, emergency department. 
where $Y_{\mathrm{i}}$ represents respondent i's answer to how he/she agrees with statement $\mathrm{Y}$, with answers ranging from $1=$ Totally disagree to $5=$ Totally agree. Age $_{\mathrm{i}}$ represents the respondent's age categories divided in 5 brackets $(20-30,30-40,40-50,50-60,>60)$. Gender ${ }_{i}$ represents the respondent's sex, coded as 1 if female, 0 if male. Experience $_{\mathrm{i}}$ indicates years of experience as nurse in general, coded in 5 brackets $(<3,3-5,5-10,10-20,>20)$. Triage $\mathrm{i}_{\mathrm{i}}$ reports years of experience as triage nurse divided into 6 brackets $(\leq 1,1$ $3,3-5,5-10,10-20,>20)$.

\section{Results}

A total of 301 questionnaires were filled out and returned, with a variable number of responses to each single item (leading to a total of responses varying from 264 to 301 per item). Most of the answers came from female nurses [169 vs 95; 37 not declared]. Age distribution, number of years from degree, number of years working as a triage nurse are shown in Tables 3-5.

The overall results to each response are briefly shown in Figure 1. Table 2 reports in detail the mean values $\pm \mathrm{SD}$ of the respondents' rating of their agreement or disagreement with each of the eleven statements. Table 6 reports the results of the linear regressions for each item $\mathrm{Y}$, according to the formula described in the Materials and Methods section.

Our results indicate a substantial agreement of the triage nurses on the necessity to measure the patient's pain at triage time (item 1 ), with the most frequent answer (mode) equal to 5 (completely agree) and mean equal to 4.3. The majority declares using NRS scale to measure pain (item 2 , mode $=4$, mean $=3.8$ ), and attributing priority code based on NRS value (item 3 , mode $=4$, mean $=$ 3.4). We didn't find any significant difference in the responses with respect to age, sex, years of experience or triage for any of these items.

In general, triage nurses believe that NRS is only indicative and that their judgement matters (item 4 NRS results are only approximate. I decide the triage code based on my personal judgement, mode $=4$, mean $=3.2$ ). For this item, we found a significant difference based on gender, with females regarding the NRS reliability higher than males who instead value more their personal judgment (females 3.1 vs males 3.4; $\mathrm{P}=0.021$ uncontrolled t-test; coeff. $=-0.39, \mathrm{P}=0.015$ from regression controlling for the other factors), and a trend toward higher confidence as years of triage

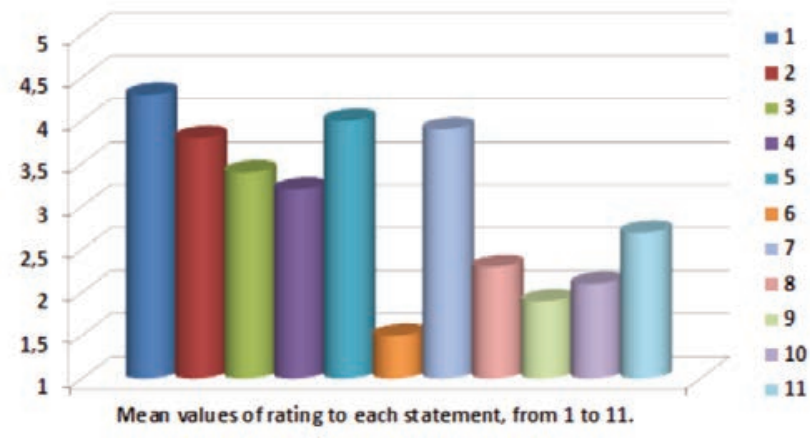

Figure 1. The figure shows the mean values of responses to each statement. experience increase (coeff. $=0.111, \mathrm{P}=0.069$ ). The vast majority of triage nurses do believe that the patients will indicate a fake higher NRS value with the aim to get a more urgent code (item 5 , mode $=$ 5 , mean $=4)$. Only a small minority, on the contrary, expects that patients would underestimate their NRS for fear of penalizing

Table 2. Mean values \pm standard deviation (SD) of rating attributed from responders to the eleven statements.

\begin{tabular}{lll} 
Question N. & Number of responsesMean value \pm SD \\
1 & 300 & $4.29 \pm 1.00$ \\
2 & 301 & $3.76 \pm 1.10$ \\
\hline 3 & 297 & $3.36 \pm 1.22$ \\
4 & 297 & $3.21 \pm 1.24$ \\
\hline 5 & 298 & $3.99 \pm 1.05$ \\
6 & 301 & $1.51 \pm 0.94$ \\
\hline 7 & 300 & $3.94 \pm 0.82$ \\
8 & 299 & $2.34 \pm 1.09$ \\
\hline 9 & 301 & $1.87 \pm 1.02$ \\
10 & 300 & $2.10 \pm 1.13$ \\
\hline 11 & 300 & $2.76 \pm 1.29$ \\
\hline
\end{tabular}

Table 3. Age classes of the responders.

\begin{tabular}{lcc} 
Agge class & N & $\%$ \\
$20-30$ & 43 & 14.3 \\
$30-40$ & 104 & 34.6 \\
\hline $40-50$ & 112 & 37.2 \\
$50-60$ & 36 & 11.9 \\
\hline$>60$ & 4 & 1.3 \\
Not declared & 2 & 0.7 \\
\hline
\end{tabular}

Table 4. Subdivision of the sample according to the number of years from degree.

\begin{tabular}{lcc} 
Years from degree & $\mathbf{N}$ & $\%$ \\
$<3$ & 15 & 4.9 \\
$3-5$ & 67 & 22.3 \\
\hline $5-10$ & 56 & 18.6 \\
$10-20$ & 76 & 25.2 \\
\hline 20 & 81 & 26.9 \\
Not declared & 6 & 2 \\
\hline
\end{tabular}

Table 5. Subdivision of the sample according to the number of years working as a triage nurse.

\begin{tabular}{lcc} 
Years working as a triage nurse & $\mathbf{N}$ & $\%$ \\
$<1$ & 46 & 15.3 \\
$1-3$ & 43 & 14.3 \\
\hline $5-5$ & 31 & 10.3 \\
$5-10$ & 82 & 27.4 \\
\hline $10-20$ & 80 & 26.6 \\
$>20$ & 17 & 5.6 \\
\hline
\end{tabular}

Not declared
0.7 
more urgent patients (item 6 , mode $=1$, mean $=1.5$ ). For this last item (Several patients declare a low NRS value due to the worry to penalize patients with worse problems), the data show a positive correlation between such belief and age categories (coeff. $=0.15$; $\mathrm{P}=0.046$ ) and a negative one with increasing categories of triage experience (coeff. $=-0,13 ; \mathrm{P}=0.002)$.

Triage nurses exhibit confidence in their ability to assess the appropriate color code based on patient suffering (item 7 , mode $=$ 4 , mean $=3.9)$ without much overestimation to avoid patient's complaints (item 8 , mode $=2$, mean $=2.3$ ) and even less underestimation for fear of overburdening the ED (item 9, mode $=1$, mean $=1.9$ ). For item 7 (I believe to give correct triage codes, appropriate to the suffering condition of the patients) we found a significant positive correlation between agreement with this statement and increasing categories of years of triage experience (coeff. $=0.09$; $\mathrm{P}=0.024$ ).

With respect to what triage nurses think about whether NRS provides a biased estimate of a patient's condition, very few believe that such scale underestimates it (item 10 , mode $=1$, mean $=2.1$ ), while the majority is ambivalent about whether such scale overestimates it (item 11, mode $=3$, mean $=2.8)$. For item $10(B y$ using NRS there is a risk of underestimate patients 'problems) we found a significant positive correlation with increasing age categories (coeff. $=0.26 ; \mathrm{P}=0.008$ ), and a significant negative one with increasing years of working experience as a nurse (coeff. $=-0.16$; $\mathrm{P}=0.022$ ). Finally, for item 11 (By using NRS there is a trend toward overestimate patients' problems), we found a significant negative correlation between agreement with this statement and increasing years of working experience as a nurse (coeff. $=-0.17$; $\mathrm{P}=0.029$ ).

\section{Discussion}

The results of this study seem to suggest that a group of Italian ENs are confident with NRS scale for pain measurement at triage time, and that the vast majority of them always use this tool. Nevertheless, there is a high percentage of nurses that consider NRS score as only suggestive, thus basing their judgement on personal experience. This seems to mainly originate from the belief that several patients will indicate an artificially higher NRS value to get a more urgent code. In fact, responses somewhat conflicting have been obtained from statements 3 to 6 .

It should be considered that patients population with a painful condition in the ED is extremely heterogeneous, and obviously different from selected patients managed in hospital pain clinics. Several cognitive, emotional, psychological, cultural and social factors, including anxiety and haste to be visited, could jeopardize the reliability of NRS in the ED.,3,13-15

The studies on this topic have reached a kaleidoscope of conclusions, sometimes non concordant or even conflicting. For example, an interesting study has shown that a visual-analogic scale, namely the Wong-Baker scale (usually used in the pediatric setting), performed better than NRS in an overcrowded ED. ${ }^{16}$ Another interesting and intriguing study, performed in Italians EDs, comparing NRS (rated 1-10) with a fake NRS (rated 30-50), has shown that the fake scale underrated pain intensity when comparing with official NRS (mean 5.5 vs 7.4). The Authors argue that, since in Italy there is an old school legacy according to which the highest value is more rewarding and 6 represents the minimum threshold, the 0-10 NRS scale could have unconsciously oriented patients to set themselves starting from a minimum psychological threshold, represented by $6 .{ }^{17}$

Moreover, since the triage nurse is usually the first health care provider to assess pain among ED patients, the time of pain relief is often dependent on the efficiency and reliability of the triage system. ${ }^{18}$ In some facilities, especially in North Europe, patientreported pain is validated by a nurse to ensure that it is neither over nor underreported. ${ }^{19}$ A recent Danish study has documented a 70\% agreement on pain category between the nurses, being the pain assessment unaffected by age, gender, or environmental issues. The Authors conclude that their findings justify that nurse-perceived pain assessment is used for triage in the emergency department. $^{20}$

One major concern has been driven by the fact that, despite declaring a high NRS score, several patients refuse analgesia delivered by the triage nurse. In an interesting study conducted in France, $38 \%$ of patients refused analgesia at triage, mainly $(53 \%)$ because Pain is bearable without analgesic. Interestingly, none of the patients were reluctant to acetaminophen delivery by a nurse. ${ }^{21}$

Table 6. Results of the linear regressions for each item $Y$, according to the following formula: $Y_{i}=\beta 0+\beta 1$ Age $i+\beta 2 G e n d e r_{i}$ $+\beta 3$ Experience $_{i}+\beta 4$ Triage $_{i}+\varepsilon i$. Where $Y i$ represents respondent i's answer to how he/she agrees with statement $Y$, with answers ranging from $1=$ Totally disagree to $5=$ Totally agree.

\begin{tabular}{|c|c|c|c|c|c|c|c|c|c|c|c|}
\hline Variables & 1 & 2 & 3 & 4 & 5 & 6 & 7 & 8 & 9 & 10 & 11 \\
\hline Age & $\begin{array}{c}-0,01 \\
(0,08)\end{array}$ & $\begin{array}{c}0,04 \\
(0,10)\end{array}$ & $\begin{array}{c}0,07 \\
(0,11)\end{array}$ & $\begin{array}{c}0,04 \\
(0,11)\end{array}$ & $\begin{array}{l}-0,05 \\
(0,09)\end{array}$ & $\begin{array}{l}0.15^{* *} \\
(0,08)\end{array}$ & $\begin{array}{c}0 \\
(0,07)\end{array}$ & $\begin{array}{c}0,06 \\
(0,10)\end{array}$ & $\begin{array}{c}-0,06 \\
(0,09)\end{array}$ & $\begin{array}{c}0.26^{* * *} \\
(0,10)\end{array}$ & $\begin{array}{c}0 \\
(0,12)\end{array}$ \\
\hline Female gender & $\begin{array}{c}-0,09 \\
(0,12)\end{array}$ & $\begin{array}{c}-0,13 \\
(0,14)\end{array}$ & $\begin{array}{c}0,02 \\
(0,16)\end{array}$ & $\begin{array}{c}-0.39 * * \\
(0,16)\end{array}$ & $\begin{array}{c}-0,05 \\
(0,13)\end{array}$ & $\begin{array}{c}-0,12 \\
(0,11)\end{array}$ & $\begin{array}{c}-0,03 \\
(0,10)\end{array}$ & $\begin{array}{c}-0,03 \\
(0,14)\end{array}$ & $\begin{array}{c}-0,1 \\
(0,13)\end{array}$ & $\begin{array}{l}-0,01 \\
(0,14)\end{array}$ & $\begin{array}{c}-0,11 \\
(0,17)\end{array}$ \\
\hline Years from degree & $\begin{array}{c}0 \\
(0,06)\end{array}$ & $\begin{array}{c}0,04 \\
(0,07)\end{array}$ & $\begin{array}{c}0,02 \\
(0,08)\end{array}$ & $\begin{array}{c}0,01 \\
(0,08)\end{array}$ & $\begin{array}{c}0,05 \\
(0,06)\end{array}$ & $\begin{array}{l}-0,03 \\
(0,05)\end{array}$ & $\begin{array}{c}-0,01 \\
(0,05)\end{array}$ & $\begin{array}{l}-0,07 \\
(0,07)\end{array}$ & $\begin{array}{c}-0,02 \\
(0,06)\end{array}$ & $\begin{array}{c}-0.16^{* *} \\
(0,07)\end{array}$ & $\begin{array}{c}-0.17^{* *} \\
(0,08)\end{array}$ \\
\hline Years working as a triage nurse & $\begin{array}{c}-0,02 \\
(0,05)\end{array}$ & $\begin{array}{c}0 \\
(0,05)\end{array}$ & $\begin{array}{c}-0,1 \\
(0,06)\end{array}$ & $\begin{array}{l}0.11^{*} \\
(0,06)\end{array}$ & $\begin{array}{c}0,06 \\
(0,05)\end{array}$ & $\begin{array}{c}-0.13^{* * *} \\
(0,04)\end{array}$ & $\begin{array}{l}0.09^{* *} \\
(0,04)\end{array}$ & $\begin{array}{c}0 \\
(0,05)\end{array}$ & $\begin{array}{l}-0,03 \\
(0,05)\end{array}$ & $\begin{array}{c}-0,02 \\
(0,05)\end{array}$ & $\begin{array}{c}0,08 \\
(0,06)\end{array}$ \\
\hline Constant & $\begin{array}{c}4.53^{* * *} \\
(0,20)\end{array}$ & $\begin{array}{c}3.61^{* * *} \\
(0,24)\end{array}$ & $\begin{array}{c}3.41^{* * *} \\
(0,26)\end{array}$ & $\begin{array}{c}2.91^{* * *} \\
(0,26)\end{array}$ & $\begin{array}{c}3.81^{* * *} \\
(0,22)\end{array}$ & $\begin{array}{c}1.71^{* * * *} \\
(0,18)\end{array}$ & $\begin{array}{c}3.76^{* * * *} \\
(0,16)\end{array}$ & $\begin{array}{c}2.43^{* * * *} \\
(0,23)\end{array}$ & $\begin{array}{c}2.22 * * * \\
(0,22)\end{array}$ & $\begin{array}{c}2.01^{* * *} \\
(0,24)\end{array}$ & $\begin{array}{c}3.09 * * * \\
(0,28)\end{array}$ \\
\hline Observations & 259 & 260 & 257 & 257 & 257 & 260 & 259 & 260 & 260 & 259 & 259 \\
\hline R-squared & 0 & 0,01 & 0,01 & 0,05 & 0,01 & 0,04 & 0,03 & 0,01 & 0,01 & 0,03 & 0,02 \\
\hline
\end{tabular}

Standard errors in brackets. ${ }^{* * *} \mathrm{P}<0.01,{ }^{* *} \mathrm{P}<0.05,{ }^{*} \mathrm{P}<0.1$. 
It seems, thus, that the expressed need for analgesia could be more reliable than NRS in initial management of acute pain in the ED. Moreover, it has been documented that after implementation of mandatory documentation regarding pain assessment in the ED, despite a significant increase of analgesics delivery, patients' satisfaction with pain management did not significantly increase. ${ }^{22}$

We think that these data could at least in part rely on the different utilizations of NRS. In post-operative setting, for example, the tool is used to drive analgesic dosing and administration, while at ED's triage NRS is mainly used to attribute priority code. This represents, obviously, a substantial and biasing difference. The aforementioned skepticism has led to a widespread tendency to abandon NRS as a reliable tool in the US EDs. ${ }^{23}$ As a consequence, some Authors have recently argued that a pain protocol, based on administration of analgesic agents, driven solely by patient response to a simple standardized question repeated at intervals, resulted in achievement of satisfactory analgesia in the vast majority of traumatic as well as non-traumatic painful conditions, and could be preferable to the use of pain rating scales. ${ }^{24-27}$

Main limitations of this study are the relatively small sample and the fact that questionnaire deals with subjective perceptions. Otherwise, the concept of perception is declared yet in the title.

\section{Conclusions}

In conclusion, NRS confirms to be a potentially valuable tool for pain evaluation at triage time, but many nurses express some doubts on its reliability, and will attribute the triage code mainly basing on their own judgement. Further studies are needed to assess and validate new tools for pain measurement at triage time in the EDs.

\section{References}

1. Chapman CR. Measurement of pain: problems and issues. In: Bonica JJ, Albe-Fessard D, eds. Advances in pain research and therapy, Vol. 1. New York: Raven Press, 1976. pp 345-353.

2. Jensen MP, Karoly P, O'Riordan EF, et al. The subjective experience of acute pain. An assessment of the utility of 10 indices. Clin J Pain 1989;5:153-159.

3. Morley S. Psychology of pain. Br J Anaesth 2008;101:25-31.

4. Marco CA, Nagel J, Klink E, et al. Factors associated with self-reported pain scores among ED patients. Am J Emerg Med 2012;30:331-7.

5. Todd KH. Pain assessment instruments for use in the emergency department. Emerg Med Clin N Am 2005;23:285-95.

6. Morone NE, Weiner DK. Pain as the 5th vital sign: exposing the vital need for pain education. Clin Ther 2013;35:1728-32.

7. Berthier F, Potel G, Leconte P, et al. Comparative study of methods of measuring acute pain intensity in an ED. Am J Emerg Med 1998;16:132-6.

8. Bijur PE, Latimer CT, Gallagher EJ. Validation of a verbally administered numerical rating scale of acute pain for use in the emergency department. Acad Emerg Med 2003;10:390-2.

9. Seymour RA. The use of pain scales in assessing the efficacy of analgesics in post-operative dental pain. Eur J Clin Pharmacol 1982;23:441-4.

10. Ekblom A, Hansson P. Pain intensity measurements in patients with acute pain receiving afferent stimulation. J Neurol Neurosurg Psychiatr 1988;51:481-6.
11. Wilkie D, Lovejoy N, Dodd M, et al. Cancer pain intensity measurement: concurrent validity of three tools - finger dynamometer, painintensity number scale, visual analogue scale. Hosp J 1990;6:1-13.

12. Paice J, Cohen F. Validity of a verbally administered numeric rating scale to measure cancer pain intensity. Cancer Nurs 1997;20:88-93.

13. Motov SM, Khan AN. Problems and barriers of pain management in the emergency department: Are we ever going to get better? J Pain Res 2008;2:5-11.

14. Todd KH, Ducharme J, Choiniere M, et al; PEMI Study Group. Pain in the emergency department: results of the pain and emergency medicine initiative (PEMI) multicenter study. J Pain 2007;8:460-6.

15. Craven P, Cinar O, Madsen T. Patient anxiety may influence the efficacy of ED pain management. Amer J Emerg Med 2013;31:313-8.

16. Riccardi A, De Iaco F, Guiddo G, et al. The use of a Wong baker scale in the assessment of acute pain in adults in the overcrowded environment of the ED. Ital J Emerg Med 2015;4:8-12.

17. Capponi R, Loguercio V, Guerrini S, et al. Does the numeric rating scale (NRS) represent the optimal tool for evaluating pain in the triage process of patients presenting to the ED? Results of a multicenter study. Acta Biomed 2016;87:347-52.

18. Ducharme J1, Tanabe P, Homel P, et al. The influence of triage systems and triage scores on timeliness of ED analgesic administration. Am J Emerg Med 2008;26:867-73.

19. Lindberg SO, Lerche la Cour J, Folkestad L, et al. The use of triage in Danish emergency departments. Dan Med Bull 2011;58:A4301.

20. Hangaard MH, Malling B, Mogensen CB. High inter-observer agreement of observer-perceived pain assessment in the emergency department. BMC Emerg Med 2018;18:8.

21. Lecomte F, Audureau E, Guyerdet V, et al. Patients in pain that refuse acetaminophen at triage. Am J Emerg Med 2014;32:388-9.

22. Sturesson L, Falk AC, Castrén M, et al. Mandatory documentation of pain in the emergency department increases analgesic administration but does not improve patients' satisfaction of pain management. Scand J Pain 2016;13:32-5.

23. Levy N, Sturgess J, Mills P. "Pain as the fifth vital sign" and dependence on the "numerical pain scale" is being abandoned in the US: Why? Brit J Anaesth 2018;120:435-8.

24. Chang AK, Bijur PE, Holden L, et al. Efficacy of an Acute pain titration protocol driven by patient response to a simple query: do you want more pain medication? Ann Emerg Med 2016;67:565-72.

25. Smith JE, Rockett M, Creanor S, et al. PAin SoluTions In the Emergency Setting (PASTIES) - patient controlled analgesia versus routine care in emergency department patients with non-traumatic abdominal pain: randomised trial. BMJ 2015;350:h3147.

26. Smith JE, Rockett M, Squire R, et al. PAin SoluTions In the Emergency Setting (PASTIES)-patient controlled analgesia versus routine care in emergency department patients with pain from traumatic injuries: randomised trial. BMJ 2015;350:h2988.

27. Rahman NH, DeSilva T. A randomized controlled trial of patient-controlled analgesia compared with boluses of analgesia for the control of acute traumatic pain in the emergency department. J Emerg Med 2012;43:951-7. 